\title{
Basics of seeing motion
}

\author{
Fundamentos da visão de movimento
}

Walter H.Ehrenstein

Correspondence concerning this article should be addressed to Walter H. Ehrenstein, Leibniz Research Center for Working Environment and Human Factors, University of Dortmund, ArdeystraBe 67 - D - 44139, Dortmund, Germany (email: ehrenst@arb-phys.uni-dortmund.de).

\section{ABST RACT}

This chapter is about basic conditions of seeing motion (mostly of single objects in the frontoparallel plane). It emphasizes the richness and challenge of phenomena in motion perception. Apart from the distinction between afferent (retinal) and efferent (oculomotor) mechanisms, motion percepts can result from real (object) motion as well as from various forms of apparent motion (stroboscopic or phi motion, induced motion, autokinesis). Further intriguing motion phenomena include motion contrast and assimilation, motion adaptation and aftereffects, and velocity transposition. Finally, anticipatory performance of motion extrapolation is considered as a dynamic case of amodal perceptual completion, indicating that visual motion is closely related to action.

Keywords: Motion perception; Afferent/efferent mechanisms; Oculomotor system; Apparent motion; Velocity transposition; Motion extrapolation

\section{INTRODUCTION}

Motion is perhaps the most important and powerful visual stimulus dimension. Practically everything of any interest in the visual world moves. Stimuli that share approximately the same speed and direction are seen as a unit, a figure, that is easily segregated from the background according to the Gestalt factor of common fate ${ }^{(1)}$. For example, an animal that is perfectly camouflaged as long as it remains still, will pop out as soon as it moves. Motion can tie together stimuli that are widely distributed over the visual field, by this overriding proximity and similarity as perceptual grouping factors.

Even when the objects around us are stationary, their images on the retina can move, because the eyes and head are never entirely still. It is therefore not surprising to learn that separate visual subsystems exist that are specialized to process motion as there are subsystems mediating the perception of brightness, form, or color ${ }^{(2-4)}$.

Another aspect of motion is that it is closer than any static stimulus dimension tied to motor action. Motion signals a change in the conditions of the environment, and change may require reaction. It may mean the approach of danger or of a desirable target.

If we just consider the motion of a single object we can distinguish absolute from relative motion. Absolute motion perception refers to the case of an object which is seen in an otherwise homogenous field (Ganzfeld), or in a field in which the object is so far removed from other objects that their presence does not affect its judgement. The other, much more common, form of motion perception may be termed relative motion perception. It arises when the observer judges the motion of one object in reference to the position of other objects in her or his visual field. This chapter will focus on the basic conditions of visual motion (mostly of single objects moving in the frontoparallel plane) rather than on more complex forms of multi-element 
motion as represented by optic flow fields, biological motion, random-dot motion, motion perspective or motion parallax ${ }^{(5-7)}$.

Motion is a spatio-temporal event, defined as a change in spatial location over time. Target motion, thus, does not only convey dynamic properties such as direction and velocity, but also static spatial properties such as position and distance. For example, an observer can determine the position of a moving target at any given moment which may be indicated by an auditory signal ${ }^{(8)}$. The distance information under kinetic conditions might resemble but also differ from that under static conditions, as has been demonstrated, for example, with kinetic versions of geometrical-optical illusions ${ }^{(9)}$ or with a distance-analog of Runeson's velocity effect ${ }^{(10)}$.

Retinal image motion can result from displacement either of the object relative to a stationary environment, or of the observer relative to a stationary object. Both object and observer can also move together. All these conditions cause changes in the pattern of stimulation at the retina, but despite these we retain an appropriate representation of both our position in space as well as that of the object. Occasionally, errors do occur in our perception of motion, and these can be very instructive in understanding the nature of motion perception.

For instance, illusory percepts of motion can arise from static patterns. Striking examples of apparent motion arising from a stationary black-and-white patterns are given in Fig. 1. Most observers see a "sliding motion" of the inset relative to the surround. When the figure is slowly moved, the inner disk appears to float relative to the concentric annulus. Eye movements cannot account for this illusion, since it persists in stabilized vision where apparent motion is perceived not only for the disk, but also for the ring ${ }^{(11)}$. This illusion is an example of intrafigural apparent motion ${ }^{(12-13)}$ and is possibly based on the modulating activity of slow hyperpolarizing potentials of "configural units" in cortical area $\mathrm{V} 4^{(14)}$.

Not only do we perceive a structured meaningful world, but we see it composed of distinct objects some stationary, others moving in various directions at different rates of speed. An initial answer that one might think of is that changes in displacement of an image on the retina are detected. In fact, many cortical cells are sensitive to motion across the retina and thus could signal such changes. However, a problem arises with this simple account if you consider self-motion of the observer in the environment ${ }^{(15)}$. Changes in retinal location can be due either to objects moving in the environment or to movements of the observer. How the perceptual system resolves the locus of motion constitutes a primary problem of motion research ${ }^{(6)}$.

\section{AFFERENT ANDEFFERENT MOTION SIGNALS}

If we keep our eyes fixed, motion is perceived by the successive stimulation of adjacent retinal loci (afferent motion signals). Retinal image motion is, however, not the only condition of motion perception. When our eyes pursue a moving object, the retinal image of the pursued target is held relatively

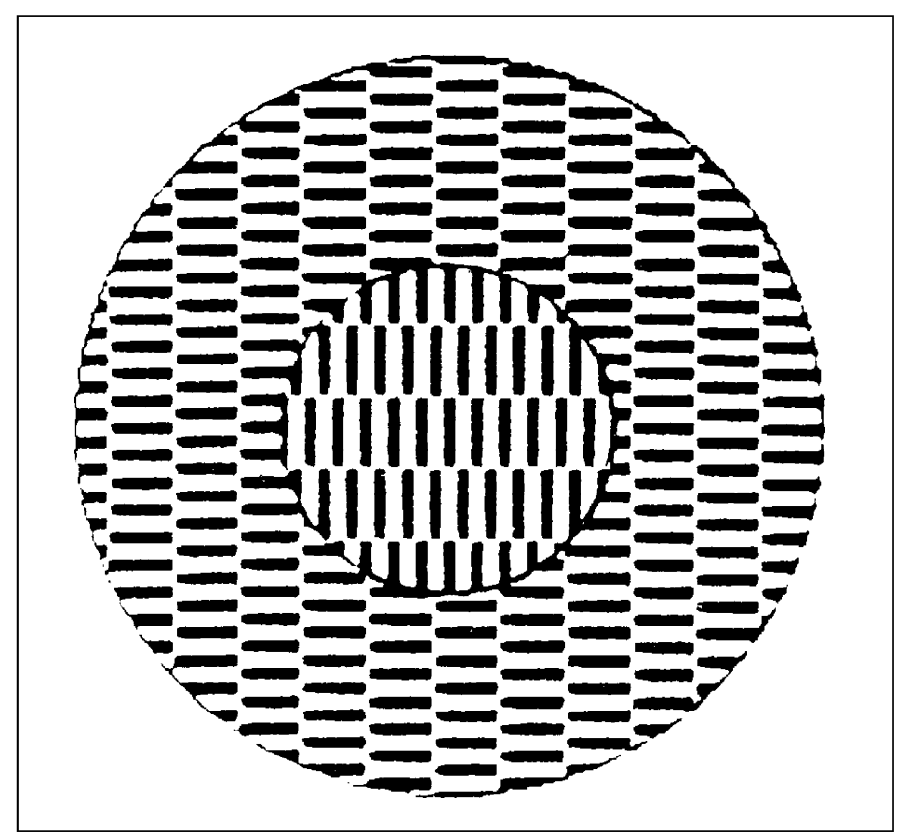

Fig. 1. Pattern for the demonstration of intrafigural apparent motion ${ }^{(11)}$. The circular inset will spontaneously appear to "move" with respect to the surrounding checkerboard, perceived either as an aperture or an occluding figure

stationary on the retina. Nevertheless, we perceive motion. Thus, the perception of motion can be also based on a sensorimotor or efferent motion signals. Afferent motion processing relies on an inflow of visual motion signals based on retinal projections of object motion. Conversely, the efferent process infers motion from the pursuit movements of the eyes following a stimulus and keeping it fixed within the fovea (Fig. 2).

The displacement of position on the retina is not sufficient to specify that an object is moving, because the displacement may be due to (eye) movements of the observer. Two theories have been classically proposed to account for the problem of how an observer's own movements are distinguished from motion in the world. The inflow theory supposes that the feedback from the muscles that control eye movements is monitored by the brain. The change due to the eye movements is then subtracted from the shift in location of the image on the retina. In contrast, outflow theory states that the motor signal sent to the eyes is monitored. A copy of this outgoing signal, which is called an efference copy or corollary discharge, is used to cancel the resulting movement of the image on the retina. This is achieved by a still hypothetical brain mechanism, a comparator, which is supposed to match the signals from the oculomotor centers with those from the afferent flow of images from the retina.

Modern research favors outflow over inflow theory ${ }^{(16)}$. An initial evidence for outflow theory is that the visual world appears to move when you press on the lid of the eye with a finger. In this case, movement is incorrectly seen because there is no efference copy associated with the commands of the eye muscles to indicate that the eye moved. Furthermore, it has been 
shown that such apparent movement can occur even when the eye actually remains stable on a fixated target, with the eye muscles providing force to prevent the finger pressure from moving the eye ${ }^{(17)}$. In this situation, illusory motion is seen because there is an efference copy but no movement of the eye. Further evidence supporting the outflow theory comes from studies in which an observer's eye muscles were temporarily paralyzed. When the observer tries to move her or his eyes (which do not actually move), the scene appears to move to a new position ${ }^{(16,18)}$.

A comprehensive functional model which supposes a reference signal that combines oculomotor information (efference copy) with optokinetic-vestibular information about head movement has been worked out by Wertheim ${ }^{(19)}$. The reference signal encodes how the eye move in space rather than in their orbits. It is matched with the retinal signal in order to distinguish percepts of object and ego motion in space. Wertheim's model can especially account for the loss of position constancy, the Filehne illusion, occurring during pursuit eye movements. There is experimental support for the model's predictions on how to manipulate the optokinetic (visual) input to change the strength of the Filehne illusion, or even invert $i^{(20-}$ 21). According to these and other findings ${ }^{(22)}$, the optokinetic pattern must be large, not move too fast, and remain visible for some time in order to ensure perceptual constancy. Although Wertheim's model addresses smooth self-motion and continuous eye movements, it may be extended to ballistic bodily movements and saccadic eye movements as well ${ }^{(23)}$.

\section{REALOR OBJ ECT MOTION}

Real or object motion means that an object is continuously displaced from one point in the outer space to another. Object motion can cause a corresponding retinal image shift, if we keep our eyes fixed. It is, however, also perceived with pursuing eyes, although in that case the image of the moving object remains stationary on the retina. Both modes of motion detection are often intermixed, for example, motion across the retina provides the stimulus for initiating pursuit eye movements.

Much of the research on real motion has focused on determining the factors which influence our threshold for motion perception under steady fixation. People are good at discriminating motion as a function of changes in retinal position. Motion can be seen if a small dot moves against a stationary background at speeds as low as 0.2 degrees of visual angle per second $(\%)$. Motion sensitivity is even greater if a stationary visual reference point is present ${ }^{(24)}$. In such situations, changes as little as $0.03 \%$ s produce a motion percept.

An important difference between afferent and efferent detection of object motion is perceived velocity. A moving object seems to move more slowly when it is pursued with smooth eye movements than when it is observed with fixed eyes. This phenomenon, known as the Aubert-Fleischl effect, may be due to an efferent underregistration of the eye velo- city $^{(25)}$. Jung ${ }^{(26)}$ has pointed out the fact that angular displacement differs for afferent and efferent conditions (see Fig. 2). The angular displacement of the fovea is smaller, if it follows a moving object (Fig. 2b) than the angle of a retinal image shift produced by the same moving object (Fig. 2a), because the eye rotates around an axis through the center of the eyeball. This difference in angular extent alone, however, cannot sufficiently account for the Aubert-Fleischl effect, since it has been found to depend also on other factors such as spatial and temporal frequency of the stimulus pattern and circular vection $^{(19,27)}$.

Models of motion detection first addressed the problem how the visual system might extract information from the stimulus on the retina, i.e. afferent conditions, were based on the visual system of insects (bees, flies) whose eyes are fixed on the head. The well-known motion-detection models by Hassenstein and Reichardt propose a bilocal delayed-coincidence detector based on bidirectional cross-correlation ${ }^{(28)}$. This can serve to understand afferent motion detection in humans as well. However, as pointed out by van de Grind, Koenderink, \& van Doorn $^{(29)}$, human bilocal detectors must be unidirectional correlators to ensure that we can cope with motion transparency (i.e., the simultaneous perception of motion in different directions and layers of depth).

Van de Grind's model is depicted in Fig. 3. A succession of images produced by a moving object shifts across the retina. The motion thus registered is due to the sequential activity of receptors in the path of the shifting retinal image feeding into bilocal delayed-coincidence detectors, i.e. into neuronal units most likely of the primary visual cortex (gobal motion detec-

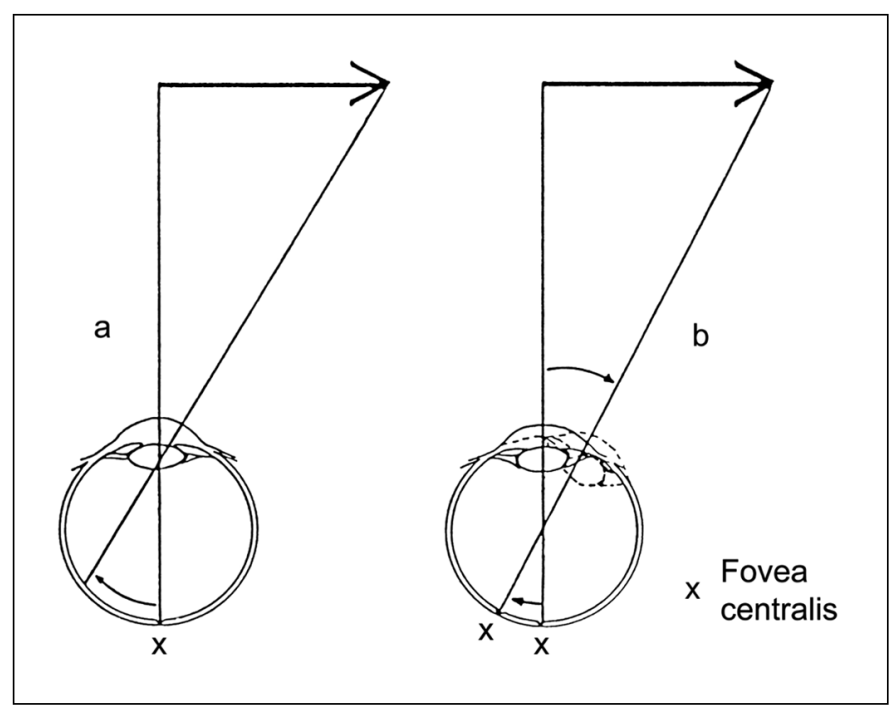

Fig. 2 - Afferent, retinal (a) and efferent, oculomotor (b) modes of motion perception ${ }^{(26)}$. a) When the eyes are held stationary, moving targets are projected with a similar angular velocity on the retina. This retinal motion leads to successive stimulation of receptive fields and motiondetection units (see Fig. 3); b) Efferently mediated motion perception occurs when the eyes pursue a moving target maintaining its retinal image in the fovea. Ocular pursuit requires a complex sensorimotor coordination and precisely controlled oculomotor innervation 
tion) and of other secondary visual cortex-representations, namely to medial temporal (MT) area and to the medial superior temporal (MST) area ${ }^{(27,31)}$. Those detectors are tuned to a given velocity $\mathrm{S} / \mathrm{T}$, where $\mathrm{S}$ is the span between two receptive fields on the retina and $\mathrm{T}$ the delay. A two-fold coincidence unit $(\mathrm{C} 2)$ receives the input from both fields and multiplies them together. The coincidence unit responds optimally when the same pattern of light strikes first the region sampled by F1 and then, some time (T) later strikes the region sampled by F2. One good stimulus would be an image that moved continuously across the retina from left to right. It is important to realize that this is not the only stimulus that would work. For example, a stationary light flashed briefly on F1 and after $\mathrm{T}$ on F2 should be as effective. We will see in the next section that indeed such nonmoving (stroboscopic) stimuli dupe the visual system into treating them as though they had moved. Thus, the basic idea in this model is that a relatively precise temporal coincidence of the signals from the subfields is required at the multiplier if it is to generate a response. It should be noted that the delay box in Fig. 3 should not taken literally to mean that this box represents a separate neuronal unit; the delay could be as well a functional part of "filter" $\mathrm{F} 1$ or be in addition reflected by the "multiplier" $\mathrm{C} 2{ }^{(29)}$.

\section{APPARENT MOTION}

The term apparent motion refers to any motion percept occurring when there is no real or object motion correspon-

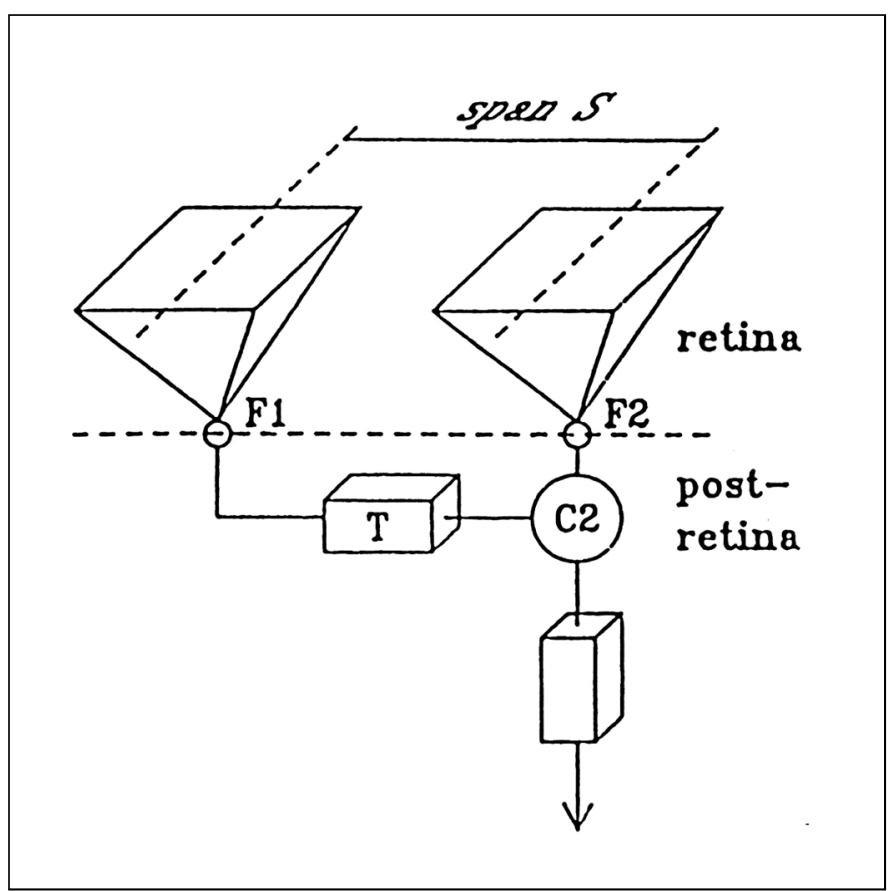

Fig. 3 - Bilocal motion detector ${ }^{(30)}$. The detector is tuned to the velocity $\mathrm{S} / \mathrm{T}$, where $\mathrm{S}$ is the span and $\mathrm{T}$ the delay. A two-fold coincidence unit (C2) receives the input from receptive fields (F1, F2) in that the delay $T$ is the difference between response latency of the pathway from $F 1$ to $\mathrm{C} 2$ and the latency from F2 to $\mathrm{C} 2$ ding to the percept. Apparent motion comprises various phenomena such as stroboscopic motion, induced motion, autokinesis, the Filehne illusion, and motion aftereffects.

\section{Stroboscopic motion}

The most prominent example of apparent motion is stroboscopic motion so that one often finds the term apparent motion just referring to this form of motion. Stroboscopic motion consists of discrete, successive changes of stimulus positions which lead to the perception of continuous motion. It is the basis for perceived motion in movies (cinema). The fact that we perceive smooth motion from motion pictures conveys the power of this form of apparent motion. Much work on stroboscopic motion has been conducted with simple displays. It can be demonstrated by just two stimuli (see Fig. 4). As the light in position $A$ flashes on and off, the light in position $B$ flashes off and on, i.e. one light is onset shortly after the other is offset.

If the spatiotemporal stimulus conditions are appropriate one perceives motion across the intervening space between $A$ and $B$ in the direction from $A$ to $B$, i.e., a single light is seen moving through the empty space between $A$ and $B$. According to Wertheimer (1912) who first systematically investigated stroboscopic motion, we can distinguish several stages. If the time interval is too short leading to a high rate of alternation, simultaneity rather than apparent motion is seen, i.e., two lights are perceived flashing each at a different location. With increasing time interval, the percept changes to partial moving and "pure motion" ( $p h i$ phenomenon) to optimal motion. If the time interval is further increased, only succession will be

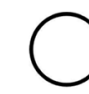<smiles>C1CCCCCC1</smiles>

Simultaneity

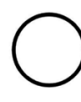<smiles>C1CCCCCC1</smiles>

Phi motion
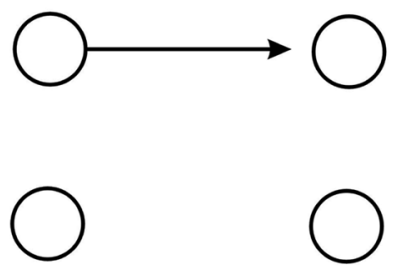

\section{Optimal motion}

a

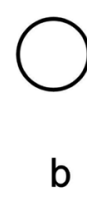

Fig. 4 - Stroboscopic motion (adapted from Wertheimer ${ }^{(32)}$ ). The perception of motion depends on the time interval between two flashing lights at a given spatial separation. With increasing time interval, the perception of two simultaneous (non-moving) lights changes to partial moving, "pure motion" (phi phenomenon) and optimally moving, to the percept of succession 
perceived - two light alternately flashing. Apparent motion can be obtained over separations of $10^{\circ}$ and more; with increasing spatial separation the temporal interval that produces optimal motion needs to be increased as well.

Strictly speaking, the term phi phenomenon should be reserved for the illusion that motion is taking place without noticeable object displacement ${ }^{(33)}$. But nowadays it is often used for all forms of stroboscopic motion and strict phi is sometimes referred to as 'pure phi'. This occurs at time intervals somewhat shorter than that necessary for optimal stroboscopic motion: one sees only two objects at their terminal positions, yet there is a clear impression of motion from one place to the other. For Wertheimer ${ }^{(32)}$ this meant that motion is just as immediate and direct a datum of experience as color or brightness; it can be perceived under appropriate conditions in a pure form, analyzable no further - an experience of sheer, objectless motion.

Further forms of stroboscopic motion have been distinguished and labelled alpha, beta, gamma, and delta motion ${ }^{(13)}$. Alpha motion refers to the case in which a line or figure is seen to expand or contract when stimuli of different sizes are presented stroboscopically. Beta motion is a synonym for optimal apparent motion, i.e., if the duration of each exposure and interstimulus interval are adequately adjusted, the appearance of motion is indistinguishable from that of a stimulus genuinely moving, hence the term optimal. Gamma motion describes the percept that a light expands for a brief time after coming on and contracts briefly when it is extinguished. Delta motion occurs when two successive stimuli are only slightly displaced from one another and the second is more intense than the first; then the second stimulus appears to move to the first.

An easy way to experience the phi phenomenon is be produced by holding a finger close enough to the eyes that double images are seen and then alternately opening and closing the two eyes: The finger seems to leap from one place to another, but no image moves across the space.

INDUCED MOTION,AUTOKINESIS

Stationary visual context, a condition mentioned before to increase motion sensitivity can also lead to illusions of motion. In such cases, motion is attributed to the wrong part of the visual display, for example, when the moon is seen racing through the clouds on a windy night. The moving clouds induce motion of the quasi-stationary moon ${ }^{(34)}$. Another example of induced motion is the experience that our train starts moving while actually another train leaves the platform. When the induced and inducing objects are in close spatial proximity, the effect is referred to as motion contrast (see section 6), whereas induced motion typically refers to conditions in which test and inducing objects are spatially separated.

An easy experimental demonstration of induced motion is a luminous stationary dot presented inside a large, luminous diamond or circle in a dark room ${ }^{(12-13,34)}$. When the circle is displaced (steadily moved or successively presented at two positions), it is the dot that will normally be seen to move in opposite direction (Fig. 5). Under both real and stroboscopic motion conditions, one of two stimuli needs to be larger, enclosing the other. If the larger stimulus is moved, all or at least part of its motion is attributed to the smaller, enclosed stimulus. The enclosing figure is presumed to serve as a frame of reference relative to which the smaller stimulus is displaced ${ }^{(25)}$.

The fact that smaller objects are more likely to move relative to larger and enclosing stimuli might reside on ecological constraints to our perception. Usually small objects move whereas larger objects, for example, walls, trees or fields in our environment are stable. Induced motion is not restricted to linear paths. For instance, a patterned stationary disk can be induced to rotate by the rotation of a surrounding concentric patterned annulus ${ }^{(34-35)}$. A number of stimulus variables can be identified that reduce or promote induced motion. For example, induced motion diminishes when the motion of the inducing pattern increases in speed ${ }^{(36)}$. Another critical condition for induced motion is that the frame that defines the background and the enclosed stimulus should be adjacent to one another, i.e. within the same plane and distance from the observer. If the surrounding field lies too far in front of or behind the enclosed target, induced motion falls off appreciably ${ }^{(37)}$. This finding points out the complex interrelationship that exists between motion and depth perception.

Another example of apparent motion is called autokinesis. This refers to motion which is experienced when fixating on a stationary point of light in an otherwise completely darkened environment. It was first observed by Alexander von Humboldt in 1799 with respect to the apparent wandering of stars $^{(35)}$. The key condition for autokinesis to occur is the lack of another visual reference or background, i.e., absolute motion mentioned in the introduction. This spatial uncertainty makes the stationary light drift or wander irregularly after some time of inspection. Typically, the point of light appears to make small excursions, but considerable motion is sometimes noticed. Autokinetic phenomena reflect the role of eye movements and resulting efferent motion signals that produce the effect of apparent motion, especially in the absence of other visual cues that may serve as a spatial reference ${ }^{(38)}$.

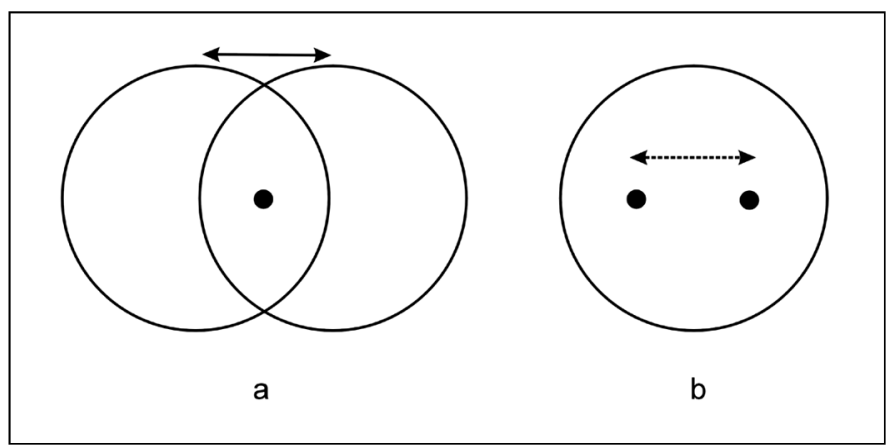

Fig. 5 - Induced motion (adapted from Ehrenstein ${ }^{(12)}$; see also Duncker ${ }^{(34)}$ ). A stationary dot appears to move in opposite direction to the actual displacement of the surrounding circle 


\section{MOTION CONTRAST AND ASSIMILATION}

When two targets travel at the same speed against a field of dots that move with a horizontal gradient of velocity, the target which is traveling faster than its immediate surround appears to have a larger absolute velocity than the target which is traveling slower than its immediate surround ${ }^{(39)}$. This phenomenon reminds on brightness contrast and is analogously referred to as motion contrast; it has been recently shown to rely likewise on lateral inhibitory interactions among specialized neuronal detectors ${ }^{(40)}$.

Conversely, an example of motion assimilation has been reported by Bressan ${ }^{(41)}$. She obtained an analog of brightness assimilation (seen in the Munker-White effect, Fig. 6a) in the velocity domain. The stimulus display consisted of two sets (separated by $9.2^{\circ}$ ) of three horizontal rows each (Fig. 6b).

There were 11 dots in each row, at a distance of $1.5^{\circ}$ from each other, and $0.6^{\circ}$ separated neighboring rows within a set. Within one set of three rows, the dots of the first and third rows (flanking rows) had the same color and moved with the same velocity $a$. The central row consisted of three separate sections; the dots of the left and right sections (collinear rows, 7 dots each) had the same color as the dots of the flanking rows and moved with velocity $b$; the dots of the central section (test row, 4 dots) had a different color and moved with velocity $c$. The upper and lower sets were identical except for the fact that assignments of velocities to the contextual rows would always be reversed: so if flanking and collinear rows traveled respectively with velocities $a$ and $b$ in the upper set, they would travel respectively with velocities $b$ and $a$ in the lower set, and vice versa. Dots moved horizontally at a constant speed across the screen; whenever a dot disappeared at one end of the screen a new dot appeared on the other end, producing a continuous stream of moving elements. Velocities $a$ and $b$ were fixed at $3^{\circ}$ and $9 \%$, respectively. The velocities of the test rows were varied across trials, and were either $6 \%$ or slightly more or less.

Observers had to compare the apparent velocity of the two test rows and to report which row appeared to be moving faster. Under these conditions, the test row flanked by highspeed rows (Fig. 6b, upper set) looked faster not only when it actually was as fast as the other, flanked by low-speed rows (Fig. 6b, lower set), but even when it was actually slower ${ }^{(41)}$. In order to be perceived as travelling at the same speed, the testdot velocity in the upper set could be by at least $0.6 \%$ slower than in the lower set.

\section{MOTION ADAPTATIONAND AFTEREFFECTS}

Prolonged inspection of a moving stimulus leads to a gradual decrease in perceived speed resulting from motion adaptation. For example, a slowly rotating spoke wheel viewed peripherally appears to slow down and ultimately come to an apparent standstill, although its physical speed is unchan$\operatorname{ged}^{(42)}$. When the speed of the wheel is slightly reduced, the perceived rotation resumes, but now in the opposite direction.

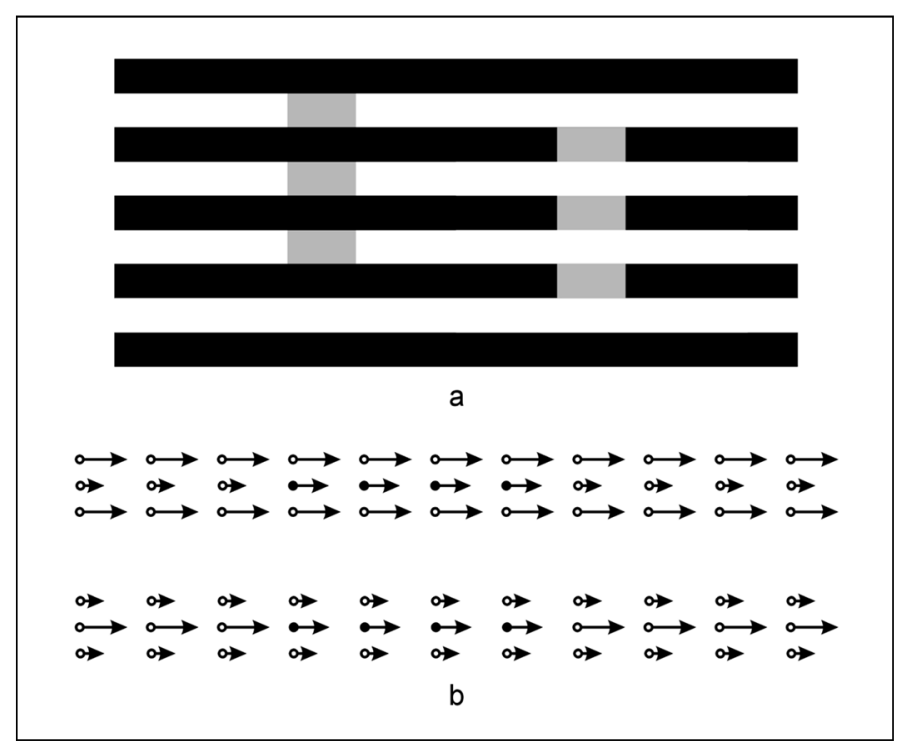

Fig. 6 - Velocity assimilation ${ }^{(41)}$. a) Brightness assimilation (MunkerWhite effect). Mid-grey patches (of identical luminane) appear darker on the left and lighter on the right, respectively; b) Bressan effect. The arrows represent the stimulus velocities. Although the solid dots of the upper set and of the lower set are moving with the same physical speed, those in the upper set appear to be moving faster than those in the lower set. (This effect can be regarded as a velocity domain analogue of the Munker-White effect)

The subsequent effects of motion adaptation can be vividly observed if you stare at a waterfall for some time and then look at a stationary scene, for example, the adjacent rocks. The scene will appear to be moving upward. This is called the waterfall illusion and is a striking example of a motion aftereffect. A likely first explanation of the waterfall effect first proposed by Addams in 1834 was that the downward pursuit movements established in response to the water persisted when the eyes were turned to the rocks ${ }^{(43)}$. However, a similar aftereffect is obtained if you watch a rotating spiral (Fig. 7). If the spiral appears to shrink or recede during rotation, it will appear to expand or approach when the rotation is suddenly stopped.

It is obvious that no sort of eye movements can account for the fact that an object seems to expand in all directions at once. Furthermore, as first demonstrated by Mach and Dvorák in $1870^{(44)}$, it is impossible for the eyes to shrink and expand at the same time as do the seen afterimages when adapting to the rotation of two concentrically arranged spirals of opposite throw, see Fig. 7.

Even for the waterfall illusion, the eye-movement explanation is inadequate, since one can obtain the same effect, at least in part of your visual field, if one fixates a point at the rock at one side of the fall, in which case pursuit eye movements are excluded ${ }^{(45)}$.

Motion aftereffects typically consist on illusory motion in opposite direction to previous exposure to continuous motion in the same direction. They provide evidence that motion perception is not merely bound on stimulus motion and indicate the dynamics of special direction-sensitive motion-units 


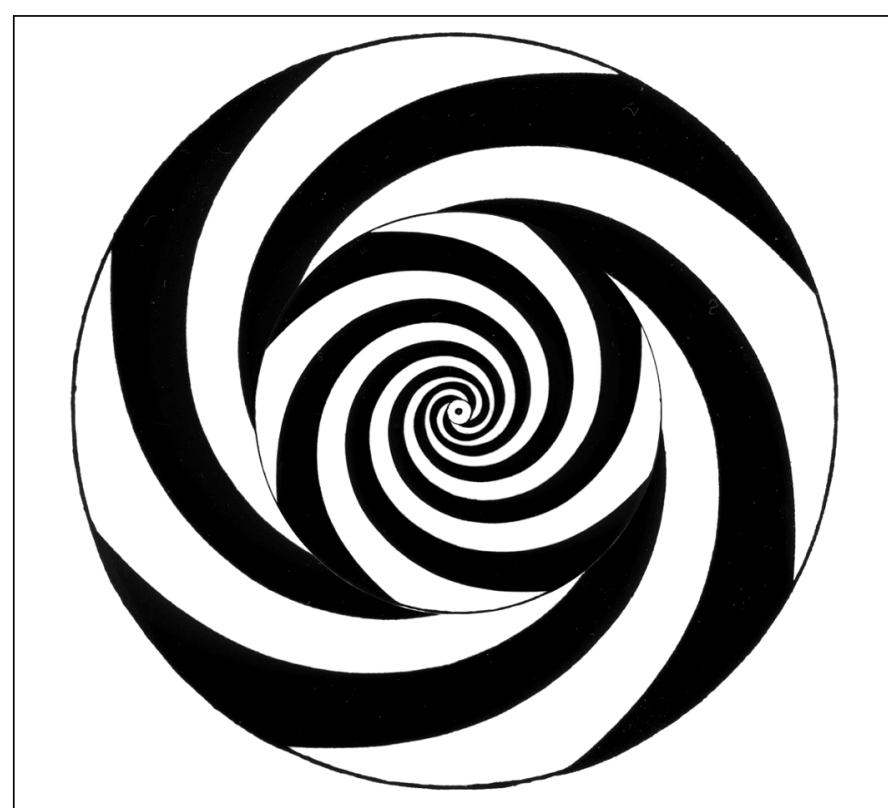

Fig. 7 - Spiral aftereffect (adapted from Dvorák (1870), see Broerse, Dodwell, \& Ehrenstein $\left.{ }^{(44)}\right)$. Successive motion contrast in opposite direction is observed after rotation of the depicted spiral pattern. It consists of two concentrically organized sets spirals, a left-throw spiral superimposed on a right-throw spiral. During rotation this spiral pattern will simultaneously induce opposite, expanding and contracting, motion aftereffects in retinally adjacent areas (inspection time of $30 \mathrm{~s})$. This demonstration strikingly disproves any eye-movement account of motion aftereffects. The eyes cannot execute movements of expansion and contraction at the same time

which are selectively adapted during stimulation. Cells that selectively adapt to the direction of motion have been found in monkey area $\mathrm{MT}^{(46)}$. When stimulated by a pattern moving in their preferred direction, these cells show a brief burst of excitation at motion onset, followed by a rapid decrease of the firing rate and finally an "off" response at motion offset that falls below the resting discharge which might be a correlate of the seen aftereffect. Electrophysiological and psychophysical correlates of the motion aftereffect have also been comparatively studied in $\operatorname{man}^{(47)}$.

Interestingly, no motion aftereffect occurs following the stimulation of the entire retina by linear motion ${ }^{(48)}$. In this case, however, a displacement of sound opposite to the direction of visual motion adaptation can be observed, suggesting a cross-modal, visual-auditory aftereffect ${ }^{(49)}$. Motion aftereffects can also be obtained from (and interact with) induced motion $^{(36)}$. These and other finding $\mathrm{s}^{(50)}$ indicate the existence of several different motion aftereffects, depending upon the site at which adaptation effects occur. These aftereffects may serve as a tool for investigation of putative adaptation sites and sensory mechanisms.

\section{MOTION AND SPACE:VELOCITY TRANSPOSITION}

The size of the target in proportion to the size of the background affects strikingly its perceived speed. This phe- nomenon is called velocity transposition ${ }^{(51-52)}$ and can be regarded as a form of motion constancy ${ }^{(53)}$. It takes into account that the retinal image velocity, like retinal image extent, is inversely proportional to the distance of the imaged object from the observer. Velocity transposition can be demonstrated most simply by using two squares, each of which is moved behind a separate aperture with the size proportion of the squares and their apertures being 1:2 (see Fig. 8).

Observes, asked to adjust the velocity of one display until it appears to match that of the other, typically give an equality match when the physical speed of movement of the large aperture is set at almost twice the speed of the motion of the small aperture $^{(25,52)}$. This general effect of target size and its background on perceived speed has been recently confirmed and further elaborated by Ryan \& Zanker ${ }^{(54)}$; it also extends to motion extrapolation (see section $9^{(55)}$ ). Thus, perceived velocity of an object depends on the relation of object size relative to aperture size as well as on its physical speed in that it maintains constancy of an object's velocity despite variations in its distance and corresponding retinal projections.

LINKING PER CEPTIONTOACTION:

MOTIONEXTRAPOLATION

More than any other stimulus dimension, visual motion is linked to motor action. For example, when catching a ball we do not only perceive a moving object, but also most naturally extrapolate this perceptual information to allow for advance (and hence successful) specification of corresponding motor acts. Motion extrapolation (with respect to the stimulus) or motion anticipation (with respect to the acting subject) describes a performance of a relatively simple and most common task in daily life. This performance, although it transcends stimulus-bound or modal perception, is obviously directly (perceptually) present rather than a result of indirect cognition. Thus, extrapolation seems to be directly linked to perception with which it has been found to share common properties (see below), and may hence be best considered as a form of amodal perceptual completion ${ }^{(56)}$. Sensory, modal, motion perception already entails some sort of short-term integration in sampling motion signals over time in order to derive a velocity estimate on which amodal ways of motion perception might be based in addition to higher-order integrative cognitive processes such as visuo-spatial forms of attention and working memory ${ }^{(57)}$.

A typical motion extrapolation task consists of presenting a moving object that is "occluded" or "vanishes" en route while approaching another object or observer. The task then is to press a key to indicate when the initially visually and later virtually moving object reaches the observer or the other object. For various conditions of motion presentation, linear regression is found to describe the individual performance of extrapolation $^{(58)}$ almost as appropriately as perceived veloci$\mathrm{ty}^{(46,52)}$. Motion extrapolation thus might be considered as a 


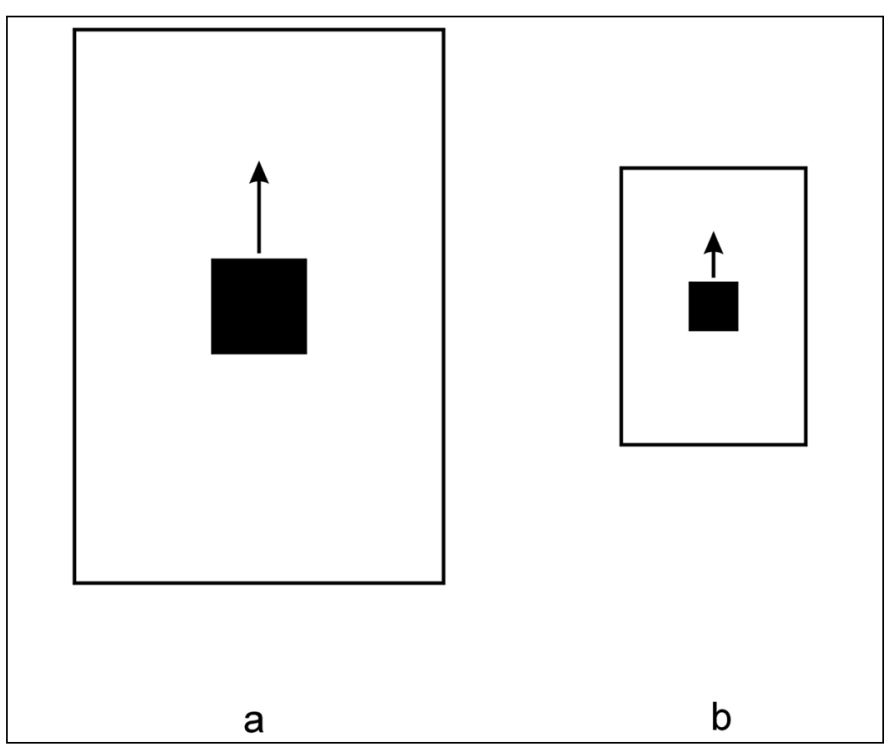

Fig. 8 - Velocity transposition (adapted from Brown ${ }^{(52)}$ ). When an observer is asked to adjust the speeds of the two squares until they match in perceived speed, the square in a must move about twice as fast than that in $b$ (motion field $a$ is double the size of motion field $b$ ). That is, if a motion field is transposed in its linear dimensions, the stimulus speeds need to be transposed in an approximately like amount for perceived velocity to appear equal

special, action-related, case of velocity perception. For example, Ehrenstein ${ }^{(59)}$ used extrapolation of visual motion to compare the performance under afferent and efferent motion conditions (see Fig. 9).

A bright dot moved horizontally over $12^{\circ}$ at $6^{\circ} / \mathrm{s}$ and then disappeared. Subjects had to extrapolate the visual motion and press a key at the moment when the virtual motion reached at one of 5 extrapolation distances, marked by LED-pairs (Fig. 9, top). In one condition, subjects had to fixate the center of the respective LED-pair from motion onset until the key press (afferent motion), in the other condition, they had to pursue the moving target with their eyes and, when it disappeared, direct their gaze to the given LED-position (efferent motion). Linear regression analysis of the mean extrapolation times (from motion offset to key press) yielded a high correlation with extrapolation distance $(r=0.99)$ in both conditions. Regression lines, however, differed in their slopes for both conditions (Fig. 9, bottom), indicating a slower extrapolation velocity for efferent $(4.2 \%)$ than afferent $(5.2 \%)$ motion conditions. This reduction in extrapolation velocity might be related to the above mentioned Aubert-Fleischl effect. The underestimation of perceived velocity of a moving object during ocular pursuit seems to extend on, or at least reflected by, motion extrapolation. Likewise, the tendency to perceive objects that move toward the observer with a shorter latency than those moving away (centripetal preference ${ }^{(60)}$ ) is also found for extrapolation in that virtual motion away from the center of fixation requires longer response times than toward the center ${ }^{(61)}$. Moreover, effects of velocity transposition are analoguously found in extrapolation tasks ${ }^{(54)}$. Thus, motion

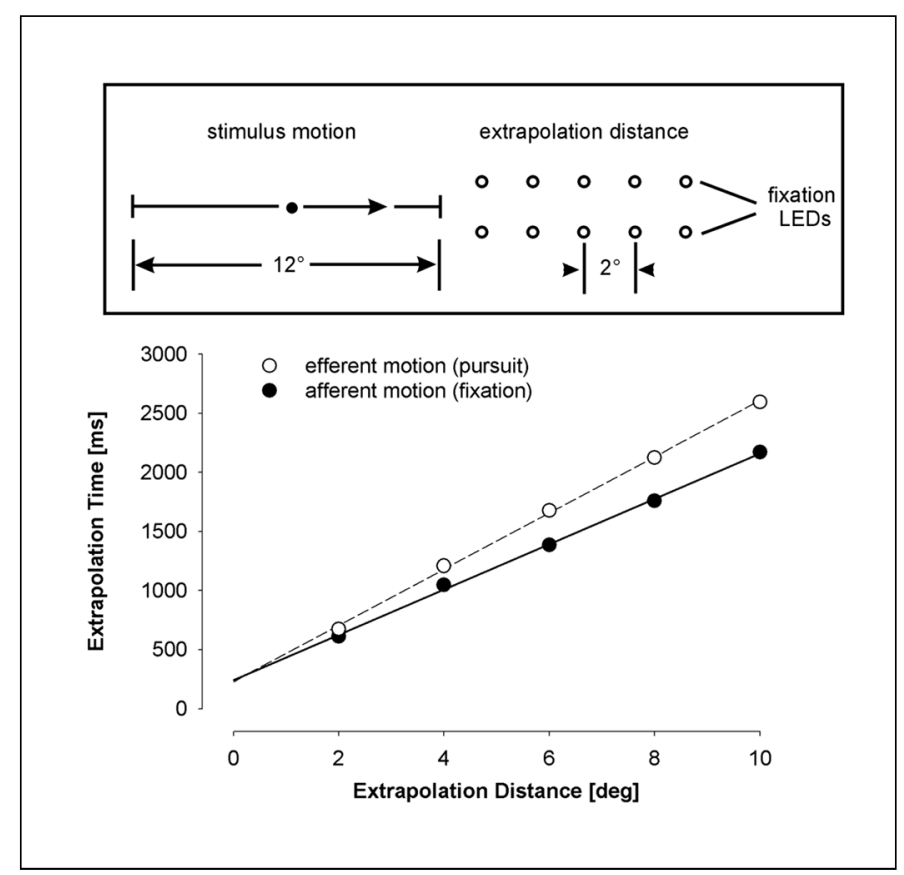

Fig. 9 - Motion extrapolation and Aubert-Fleischl effect (Ehrenstein ${ }^{(59)}$ ). A schematic view of the stimulus conditions is given on the top: A luminous dot moves at constant rate over a given distance and then disappears. Subjects are asked to press a key at the moment when the stimulus motion, if continued, would reach a position marked by one of five LED pairs (extrapolation time). In the afferent condition, fixation is between the respective target LED pair; in the efferent condition, the eyes pursue the moving dot and are then directed to the target LEDs. The resulting extrapolation times are shown below

extrapolation may be also regarded and used as an alternative method to reflect and quantify basic properties of motion perception.

\section{RESUMO}

Este capítulo é sobre as condições básicas no ver movimento (na maioria das vezes, de um único objeto no plano frontoparalelo). Ele enfatiza a riqueza e o desafio do fenômeno da percepção do movimento. Longe da distinção entre mecanismos aferente (retinal) e eferente (oculomotor), os perceptos de movimento podem resultar de movimento real (objeto) assim como de várias formas de movimento aparente (estroboscópico ou movimento phi, movimento induzido, autocinese). Outros fenômenos de movimento intrigantes incluem contraste de movimento e assimilação, adaptação e pós-efeitos de movimento, e transposição de velocidades. Finalmente, desempenho antecipatório da extrapolação do movimento é considerado como um caso dinâmico da completação perceptual amodal, indicando que o movimento visual está estreitamente ligado à ação.

Descritores: Percepção de movimento, mecanismos aferente/ eferente; Sistema oculomotor; Movimento aparente; Transposição de velocidade; Extrapolação de movimento 


\section{REFERENCES}

1. Spillmann L, Ehrenstein WH. Gestalt factors in the visual neurosciences. In: Chalupa L, Werner JS, editors, The Visual Neurosciences. Cambridge: MIT Press; 2002.

2. Zeki S. A vision of the brain. Oxford: Blackwell, 1993.

3. Spillmann L, Ehrenstein WH. From neuron to Gestalt: Mechanisms of visual perception. In: Greger R, Windhorst U, editors, Comprehensive human physiology, vol. 1. Berlin: Springer; 1996, p.861-93.

4. Sekuler R, Blake R. Perception, $4^{\text {th }}$ ed. New York: McGraw Hill, 2002.

5. Bruce V, Green PR, Georgeson M. Visual perception - physiology, psychology and ecology, $3^{\text {rd }}$ ed. Hove/Sussex: Psychology Press, 1996.

6. Wade NJ, Swanston MT. Visual perception: An introduction, $2^{\text {nd }}$ ed. Hove/ Sussex: Psychology Press, 2001.

7. Zanker JM, Zeil J. Motion vision: Computational, neural and ecological constraints. Berlin: Springer, 2001.

8. Mateeff S, Hohnsbein J, Ehrenstein WH. Visual localization and estimation of extent of target motion during ocular pursuit: A common mechanism? Perception 1990;19:459-69.

9. Stucchi N, Purghe F, Costa T. Optical-geometrical illusions in kinetic conditions. Perception 1996;25(suppl.): 136.

10. Ehrenstein WH. Perceived extent of visual motion and Runeson's effect Pflügers Arch Eur J Physiol 2001;441:R169.

11. Spillmann L, Tulunay-Keesey U, Olson J. Apparent motion in normal and stabilized vision. Invest Ophthalmol Vis Sci 1993;34(Suppl.):1031.

12. Ehrenstein W. Versuche über die Beziehungen zwischen Bewegungs- und Gestaltwahrnehmung. Z Psychol 1925;96:305-52.

13. Koffka K. Die Wahrnehmung von Bewegung. In: Bethe A, editor, Handbuch der normalen und pathologischen Physiologie, vol. XII/2. Berlin: Springer; 1931, p.1166-214.

14. Wilson HR, Krupa B, Wilkinson F. Dynamics of perceptual oscillations in form vision. Nature Neuroscience 2000;3:170-6.

15. Warren R, Wertheim AH. Perception \& control of self-motion. Hillsdale: L. Erlbaum, 1990.

16. Ebenholtz SM. Oculomotor systems and perception. Cambridge: Cambridge University Press, 2001.

17. Bridgeman B, Delgado D. Sensory effects of eyepress are due to efference. Percept Psychophys 1984;36:482-4.

18. Matin L, Picoult E, Stevens J, Edwards M, MacArthur R. Oculoparalytic illusion: Visual-field dependent spatial mislocations by humans partially paralyzed with curare. Science 1982;216:198-201.

19. Wertheim AH. Motion perception during self-motion: The direct versus inferential controversy revisited. Behav Brain Sci 1994;17:293-355.

20. Wertheim AH. Retinal and extraretinal information in movement perception: How to invert the Filehne illusion. Perception 1987;16:299-308.

21. Turano KA, Massof RW. Nonlinear contribution of eye velocity to motion perception. Vision Res 2001;41:385-95.

22. Ehrenstein WH, Mateeff S, Hohnsbein J. The strength of the Filehne illusion depends on the velocity of ocular pursuit. Perception 1990;19:411-2.

23. Bridgeman B, Blouin J. Extending reference signal theory to rapid movements. Behav Brain Sci 1994;17:315-6.

24. Palmer J. Mechanisms of displacement discrimination with a visual reference. Vision Res 1986;26:1939-47.

25. Mack A. Perceptual aspects of motion in the frontal plane. In: Boff KR, Kaufman L, Thomas JP, editors, Handbook of perception and human performance, vol. I. New York: Wiley; 1986, p.17:1-38.

26. Jung R. Einführung in die Sehphysiologie. In: Gauer OH, Kramer K, Jung R, editors, Physiologie des Menschen, vol. 13. München: Urban \& Schwarzenberg; 1978, p.1-140.

27. Pack C, Grossberg S, Mingolla E. A neural model of smooth pursuit control and motion perception by cortical area MST. J Cognit Neurosci 2001;13:102-20.

28. Reichardt W. Evaluation of optical motion by movement detectors. J Comp Physiol A Neuroethol Sens Neural Behav Physiol 1987;161:533-47.

29. van de Grind WA, Koenderink JJ, van Doorn AJ. The distribution of human motion detector properties in the monocular visual field. Vision Res 1986;26: 797-810.

30. van de Grind WA. The possible structure and role of neuronal smart mechanisms in vision. Cognit Systems 1988;2:163-80.
31. Lisberger SG, Movshon JA. Visual motion analysis for pursuit eye movements in area MT of macaque monkeys. J Neurosci 1999;19:2224-46.

32. Wertheimer M. Experimentelle Studien über das Sehen von Bewegung. Z Psychol 1912;61:161-265.

33. Steinman RM, Pizlo Z, Pizlo FJ. Phi is not beta, and why Wertheimer's discovery launched the Gestalt revolution. Vision Res 2000;40:2257-64.

34. Duncker K. Über induzierte Bewegung. Psychol Forschung 1929;12:180-259.

35. Wade NJ, Swanston MT. The representation of non-uniform motion: Induced movement. Perception 1987;16:187-94.

36. Reinhardt-Rutland AH. Induced movement in the visual modality: An overview. Psychol Bull 1988;103:57-71.

37. Gogel WC, Koslow M. The adjacency principle and induced movement. Percept Psychophys 1972;11:309-14.

38. Leibowitz HW, Shupert CL, Post RB, Dichgans J. Autokinetic drift and gaze deviation. Percept Psychophys 1983;33:455-9.

39. Loomis JM, Nakayama K. A velocity analogue of brightness contrast. Perception $1973 ; 2: 425-8$.

40. Li CY, Lei JJ, Yao HS. Shift in speed selectivity of visual cortical neurons: A neural basis of perceived motion contrast. Proc Natl Acad Sci U S A 1999; 96:4052-6.

41. Bressan P. A context-dependent illusion in the perception of velocity. Vision Res 1991;31:333-6.

42. Hunzelmann N, Spillmann L. Movement adaptation in the peripheral retina. Vision Res 1984;24:1765-9.

43. Wade NJ. A selective history of the study of visual motion aftereffects. Perception 1994;23:1111-34.

44. Broerse J, Dodwell PC, Ehrenstein WH. Experiments on the afterimages of stimulus change (Dvorák 1870): A translation with commentary. Perception 1994;23:1135-44.

45. Swanston MT. Frames of reference and motion aftereffects. Perception 1994; 23:1257-64

47. Wist ER, Diener HC, Dichgans J. Motion constancy dependent upon perceived distance and spatial-frequency of stimulus pattern. Percept Psychophys 1976;19:485-91.

46. Petersen SE, Baker JF, Allman JM. Direction-specific adaptation in area MT of the owl monkey. Brain Research 1985;346:146-50.

48. Reinhardt-Rutland AH. Aftereffect of visual movement - the role of relative movement: A review. Curr Psychol Res Rev 1987;6:275-88.

49. Ehrenstein WH, Reinhardt-Rutland AH. A cross-modal aftereffect: Auditory displacement following adaptation to visual motion. Percept Mot Skills 1996;82:23-6.

50. Wade NJ, Salvano-Pardieu V. Visual motion aftereffects: Differential adaptation and test stimulation. Vision Res 1998;38:573-8.

51. Brown JF. Über gesehene Geschwindigkeiten. Psychol Forschung 1928;10: 84-101.

52. Brown JF. The visual perception of velocity. Psychol Forschung 1931;14: 192-232.

53. Wist ER, Gross JD, Niedeggen M. Motion aftereffects with random-dot checker-board kinematograms: Relation between psychophysical and VEP measures. Perception 1994;23:1155-62.

54. Ryan J, Zanker JM. What determines the perceived speed of dots moving within apertures? Exp Brain Res 2001;141:79-87.

55. Sokolov AN, Ehrenstein WH, Pavlova MA, Cavonius CR. Motion extrapolation and velocity transposition. Perception 1997;26:875-89.

56. Michotte A, Thinès G, Crabbé G. Les compléments amodaux des structures perceptives. Leuven: University Press, 1964.

57. Corbetta M, Kincade JM, Shulman GL. Neural systems for visual orienting and their relationships to spatial working memory. J Cognit Neurosci 2002; 14:508-23.

58. Yakimoff N, Mateeff S, Ehrenstein WH, Hohnsbein J. Motion extrapolation performance: A linear model approach. Hum Factors 1993;35:501-10.

59. Ehrenstein WH. Motion extrapolation and the Aubert-Fleischl phenomenon. Perception 1994;23(suppl):27b.

60. Mateeff S, Yakimoff N, Hohnsbein J, Ehrenstein WH, Bohdanecky Z, Radil T. Selective directional sensitivity in visual motion perception. Vision Res 1991;31:131-8.

61. Ehrenstein WH, Yakimoff N, Mateeff S, Hohnsbein J, Bohdanecky Z. Centripetal preference in the perception and extrapolation of visual motion. In: Blum B, editor, Channels in the visual system: Neurophysiology, psychophysics and models. London: Freund; 1991, p.257-71. 